\title{
GENERALIZED NONLINEAR QUASI-VARIATIONAL-LIKE INEQUALITIES FOR SET-VALUED MAPPINGS IN BANACH SPACES
}

\author{
Y. P. FANG, Y. J. CHO, N. J. HUANG AND S. M. KANG
}

\begin{abstract}
In this paper, we introduce and study a new class of generalized nonlinear quasivariational-like inequalities for set-valed mappings in Banach spaces. Using the KKM technique, we prove the existence and uniqueness of solution for this class of generalized nonlinear quasivariational-like inequalities for set-valued mappings in Banach spaces. Our results extend and improve some main results of Verma.
\end{abstract}

Mathematics subject classification (2000): 47H06, 49J30, $49 \mathrm{~J} 40$.

Key words and phrases: Generalized nonlinear quasi-variational-like inequality, Generalized monotone type mapping, Generalized Lipschitzian type mapping, KKM mapping.

\section{REFERENCES}

[1] R. P. AgARWAL, Y. J. CHO AND N. J. HUANG, Sensitivity analysis for strongly nonlinear quasi-variational inclusions, Appl. Math. Lett. 13(6) (2000), 19-24.

[2] Y. J. CHO, N. J. HUANG AND S. M. KANG, Random generalized set-valued strongly nonlinear implicit quasi-variational inequalities, J. Inequal. Appl. 5(5) (2000), 515-531.

[3] N. J. HUANG, Generalized nonlinear variational inclusions with noncompact valued mapping, Appl. Math. Lett. 9(3) (1996), 25-29.

[4] N. J. HuANG, M. R. BAI, Y. J. CHO AND S. M. KANG, Generalized nonlinear mixed quasi-variational inequalities, Computers Math. Appl. 40(2-3) (2000), 205-215.

[5] N. J. HuANG, Y. P. FANG AND Y. J. CHO, A new class of generalized nonlinear mixed quasi-variational inequalities in Banach spaces, Math. Inequal. Appl. (to appear).

[6] N. J. HUANG, A new completely general class of variational inclusions with noncompact valued mapping, Computers Math. Appl. 35(10) (1998), 9-14.

[7] R. U. Verma, Nonlinear variational inequalities on convex subsets of Banach spaces, Appl. Math. Lett. 10(4) (1997), 25-27.

[8] R. U. VERMA, On monotone nonlinear variational inequality problems, Comment. Math. Univ. Carolinae 39(1) (1998), 91-98.

[9] G. X. Z. YUAN, KKM Theory and Applications, Marcel Dekker, New York, 1999.

[10] K. FAN, Some properties of convex sets related to fixed point theorem, Math. Annal. 266 (1984), 519-537. 